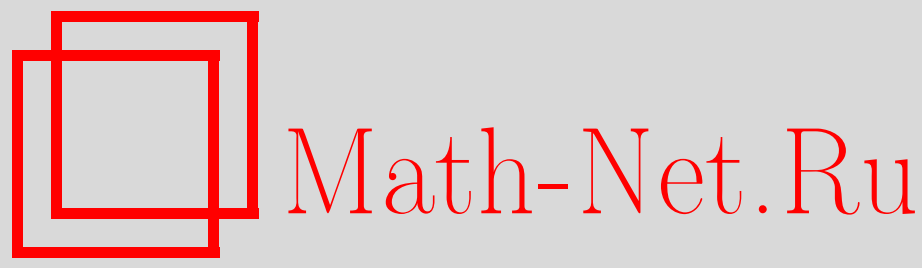

А. А. Туганбаев, Характеристические подмодули инъективных модулей над строго первичными кольцами, Дискрет. матем., 2014, том 26, выпуск 3, 121-126

DOI: https://doi.org/10.4213/dm1295

Использование Общероссийского математического портала Math-Net.Ru подразумевает, что вы прочитали и согласны с пользовательским соглашением http://www . mathnet.ru/rus/agreement

Параметры загрузки:

IP : 35.174 .16 .151

26 апреля 2023 г., 13:23:10

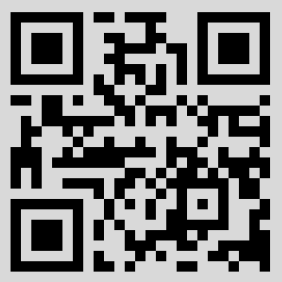




\title{
Характеристические подмодули инъективных модулей над строго первичными кольцами
}

\author{
() 2014 г. А. А. Туганбаев*
}

\begin{abstract}
Доказано, что над строго первичным справа кольцом все автоморфизм-инвариантные модули, не являющиеся сингулярными, инъективны.

Работа выполнена при поддержке РФФИ, проект 14-01-00452-А.
\end{abstract}

Ключевые слова: строго первичное кольцо, автоморфизм-инвариантный модуль, инъективный модуль, автоморфизм-продолжаемый модуль.

Все кольца предполагаются ассоциативными и с ненулевой единицей, а модули унитарными. Данная работа является продолжением работы автора [13]. Модуль называется автоморфизм-инвариантным, если он замкнут относительно автоморфизмов своей инъективной оболочки. Автоморфизм-инвариантные модули изучались в работах [2], [3], [5], [9], [10], [12], [13].

Замечание 1. В [6] кольцо называется строго первичным справа, если каждый его ненулевой идеал содержит конечное подмножество с нулевым правым аннулятором. Кольцо называется первичным, если произведение двух любых его ненулевых идеалов не равно нулю. Класс строго первичных справа колец содержит все первичные правые (или левые) кольца Голди. Свободная алгебра от двух переменных над полем является примером строго первичного справа и слева кольца, не являющегося правым или левым кольцом Голди.

Замечание 2. Из теоремы 6 [7] и теоремы 16 [3] следует, что каждый несингулярный автоморфизм-инвариантный правый модуль над первичным правым кольцом Голди является инъективным модулем. В теореме 1 из [13] доказано, что над первичным правым кольцом Голди каждый автоморфизм-инвариантный правый модуль, не являющийся сингулярным, является инъективным.

В связи с замечаниями 1 и 2 мы докажем теорему 1 , которая является основным результатом данной работы.

Теорема 1. Если A - строго первичное справа кольцо, то каждый автоморфизм-инвариантный правый $A$-модуль, не являющийся сингулярным, является ингективным.

* Место работы: Российский экономический университет имени Г.В. Плеханова, e-mail: tuganbaev@gmail.com 
Замечание 3. Каждое строго первичное справа кольцо первично и несингулярно справа, см. [6]. Первичные кольца не обязательно строго первичны справа, поскольку существуют первичные кольца, не являющиеся несингулярными справа; см. [8]. Ясно, что класс строго первичных справа колец содержит все кольца без делителей нуля и все простые кольца.

Доказательство теоремы 1 разбито на ряд утверждений, некоторые из них представляют самостоятельный интерес. Приведем необходимые определения и обозначения.

Модуль $M$ называется инбективным относительно модуля $X$ или $X$-ингективным, если для любого подмодуля $X_{1}$ в $X$ каждый гомоморфизм $X_{1} \rightarrow M$ продолжается до гомоморфизма $X \rightarrow M$. Модуль называется инбективным, если он инъективен относительно любого модуля. Пусть $A$ - кольцо, $M-$ правый $A$-модуль, являющийся подмодулем модуля $E$. Если $M \cap E_{1} \neq 0$ для любого ненулевого подмодуля $E_{1}$ в $E$, то $M$ называется существенным подмодулем в $E$ и говорят, что $E$ - существенное расширение модуля $M$. Если $E$ - инъективный модуль и $M$ - существенный подмодуль в $E$, то $E$ называется ингективной оболочкой модуля $M$. Инъективная оболочка единственна с точностью до изоморфизма. Для любого подмножества $X$ модуля $M_{A}$ через $r(X)$ обозначается правый аннулятор $\{a \in A \mid X a=0\}$ множества $X$ в кольце $A$. Через $\operatorname{Sing}(M)$ обозначается сингулярный подмодуль модуля $M$, т.е. $\operatorname{Sing}(M)$ - подмодуль в $M$, состоящий из всех таких элементов $m \in M$, что $r(m)$ - существенный правый идеал кольца $A$. Модуль $M$ называется сингулярным (соответственно, несингулярным), если $\operatorname{Sing}(M)=M$ (соответственно, $\operatorname{Sing}(M)=0)$. Подмодуль $X$ модуля $M$ называется замкнутым в $M$, если $X=X^{\prime}$ для каждого подмодуля $X^{\prime}$ в $M$, являющегося существенным расширением модуля $X$. Модуль $M$ называется равномерным, если любые два его ненулевых подмодуля имеют ненулевое пересечение, т.е. $M$ не имеет собственных замкнутых подмодулей. Кольцо $A$ называется правым кольцом Голди, если $A$ не содержит бесконечных прямых сумм ненулевых правых идеалов и $A$ - кольцо с условием максимальности для правых аннуляторов.

Другие используемые в работе определения и базовые результаты содержатся, например, в [14] и [11].

Лемма 1 [14; 16.3,16.4]. Пусть $A$ - кольцо, $X-$ правый $A$-модуль $и\left\{Y_{i}\right\}_{i \in I}$ - некоторое множество таких модулей, что $X-Y_{i}$-ингективный модуль для каждого $i \in I$.

1) Модуль X ингективен относительно любого подмодуля каждого гомоморфного образа произвольной прямой суммы любых гомоморбных образов модулей $Y_{i}$.

2) Если модуль $X$ ингективен относителъно модуля $A_{A}$, то $X$ - ингективный модуль.

Лемма 2. Пусть $A$ - кольцо, $X$ и $Y$ - правые $A$-модули, $\left\{Y_{i}\right\}_{i \in I}$ - некоторое множество правых A-модулей $u\left\{f_{i} \in \operatorname{Hom}\left(Y_{i}, Y\right)\right\}_{i \in I}$ - некоторое множество гомоморфизмов. Кроме того, пусть $X-Y_{i}$-ингективный модуль для каждого $i \in I$.

1) Модуль $X$ инвективен относительно подмодуля $\sum_{i \in I} f_{i}\left(Y_{i}\right)$ модуля $Y$.

2) Если существует мономорфизм $A_{A} \rightarrow \sum_{i \in I} f_{i}\left(Y_{i}\right)$, то модуль $X$ ингективен.

3) Если существует мономорфизм $A_{A} \rightarrow \oplus_{i \in I} Y_{i}$, то модуль $X$ ингективен.

Лемма 2 вытекает из леммы 1. 


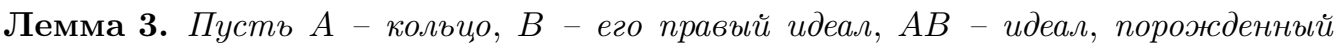
правым идеалом $B$, и $X-B_{A}$-ингективный правый $A$-модуль. Тогда:

1) $X-(A B)_{A}$-ингективный модуль.

2) Если идеал $А В$ содержит такое конечное подмножество $\left\{y_{1}, \ldots, y_{n}\right\}$, что $r\left(\left\{y_{1}, \ldots, y_{n}\right\}\right)=0$, то модуль $X$ инбективен.

Доказательство. 1) Пусть $\left\{a_{i}\right\}_{i \in I}$ - множество всех элементов кольца $A$. Для любого $i \in I$ обозначим через $f_{i}$ гомоморфизм из $B$ в $Y_{i}$, задаваемый соотношением $f_{i}(b)=a_{i} b$. Тогда $A B=\sum_{i \in I} f_{i}(B)$. По лемме $2(1) X-(A B)_{A}$-инъективный модуль.

2) Так как $r\left(y_{1}\right) \cap \ldots \cap r\left(y_{n}\right)=r\left(\left\{y_{1}, \ldots, y_{n}\right\}\right)=0$ и $y_{i} A \cong A_{A} / r\left(y_{i}\right)$ для любого $i$, то существует мономорфизм $A_{A} \rightarrow \oplus_{i=1}^{n} y_{i} A$. По лемме $2(3)$ модуль $A B$ инъективен.

Лемма 4. Пусть $A$ - кольцо и $Y$ - правый $A$-модуль, не являющийся существенным расширением сингулярного модуля. Тогда существует такой ненулевой правый идеал $B$ кольца $A$, что модуль $B_{A}$ изоморфен подмодулю модуля $Y$.

Доказательство. Так как модуль $Y$ не является существенным расширением сингулярного модуля, то существует такой элемент $y$ модуля $Y$, что $y A-$ ненулевой несингулярный модуль. Поскольку $y A \cong A_{A} / r(y)$ и модуль $y A$ несингулярен, правый идеал $r(y)$ не является существенным. Поэтому найдется такой ненулевой правый идеал $B$, что $B \cap r(y)=0$. Кроме того, существует эпиморфизм $f: A_{A} \rightarrow y A$ с ядром $r(y)$. Так как $B \cap \operatorname{Ker}(f)=0$, то $f$ индуцирует мономорфизм $g: B \rightarrow y A$. Поэтому $y A$ содержит ненулевой подмодуль $g(B)$, изоморфный модулю $B_{A}$.

Замечание 4. Модуль $M$ называется автоморфизм-продолжаемым, если каждый автоморфизм произвольного подмодуля модуля $M$ продолжается до эндоморфизма модуля $M$. Каждый автоморфизм-инвариантный модуль является автоморфизм-продолжаемым; см. [12; Лемма 3]. Кольцо целых чисел $\mathbb{Z}$ - пример автоморфизм-продолжаемого модуля, который не является автоморфизм-инвариантным.

Лемма 5. Пусть $A-$ кольцо и $M-$ автоморфизм-продолжаемый правый A-модуль.

1) Если $X$ и $Y$ - подмодули в $M$ с условием $X \cap Y=0$, причем модуль $M / X$ несингулярен, то модуль $X$ ингективен относительно $Y$.

2) Если $A$ - несингулярное справа кольцо и $Y$ - любой несингулярный подмодуль в $M$, то модуль $\operatorname{Sing}(M)$ ингективен относительно $Y$.

3) Если модуль $M$ несингулярен и $X, Y$ - замкнутые подмодули в $M$ с условием $X \cap Y=0$, то модуль $X$ ингективен относительно $Y$ и модуль $Y$ инбективен относительно $X$.

Доказательство. 1) Пусть $Y_{1}$ - подмодуль модуля $Y$ и $f_{1}: Y_{1} \rightarrow X$ - гомоморфизм. Надо доказать, что $f_{1}$ продолжается до гомоморфизма $f: Y \rightarrow X$. Без ограничения общности можно считать, что $Y_{1}$ - существенный подмодуль в $Y$. (Действительно, по лемме Цорна существует такой подмодуль $Z$ в $Y$, что $Y_{1} \cap Z=0$ и $Y_{1} \oplus Z-$ существенный подмодуль в $Y$. Обозначим $Y_{2}=Y_{1} \oplus Z$. Гомоморфизм $f_{1}: Y_{1} \rightarrow X$ продолжается до гомоморфизма $f_{2}: Y_{2} \rightarrow X$ с помощью соотношения $f_{2}\left(y_{1}+z\right)=$ $f_{1}\left(y_{1}\right)$.) 
Определим эндоморфизм $\alpha$ модуля $X \oplus Y_{1}$ соотношением $\alpha\left(x+y_{1}\right)=x+f\left(y_{1}\right)+y_{1}$ для всех $x \in X$ и $y_{1} \in Y_{1}$. Допустим, что

$$
\begin{gathered}
0=\alpha\left(x+y_{1}\right)=x+f\left(y_{1}\right)+y_{1}, \quad x \in X, y_{1} \in Y_{1}, \\
y_{1}=-x-f\left(y_{1}\right) \in X \cap Y_{1}=0, \quad f\left(y_{1}\right)=0, \\
x=x+f\left(y_{1}\right)+y_{1}=\alpha\left(x+y_{1}\right)=0 .
\end{gathered}
$$

Кроме того, для любых $x \in X$ и $y_{1} \in Y_{1}$

$$
\begin{aligned}
& x+y_{1}=\left(x-f\left(y_{1}\right)\right)+\left(f\left(y_{1}\right)+y_{1}\right)= \\
& =\alpha\left(x-f\left(y_{1}\right)\right)+\alpha\left(y_{1}\right) \in \alpha\left(X \oplus Y_{1}\right) .
\end{aligned}
$$

Поэтому $\alpha$ - автоморфизм модуля $X \oplus Y_{1}$. Из конструкции $\alpha$ ясно, что эндоморфизм $\alpha-1$ модуля $X \oplus Y_{1}$ совпадает с гомоморфизмом $f_{1}: Y_{1} \rightarrow X$ на модуле $Y_{1}$. Так как $M$ - автоморфизм-продолжаемый модуль, то автоморфизм $\alpha$ модуля $X \oplus Y_{1}$ продолжается до эндоморфизма $\beta$ модуля $M$. Обозначим через $g$ эндоморфизм $\beta-1$ модуля $M$. Тогда $g$ совпадает с $f_{1}$ на $Y_{1}$.

Докажем, что $g(y) \in X$ для любого элемента $y$ модуля $Y$. Пусть $h: M \rightarrow M / X$ - естественный эпиморфизм. Так как $Y_{1}$ - существенный подмодуль в $Y$, то $y B \subseteq$ $Y_{1}$ для некоторого существенного правого идеала $B$ кольца $A$; см., например, [4; Proposition $1.20(\mathrm{~b})]$ или $[11 ; 4.4(1)]$. Тогда

$$
\begin{gathered}
\left.g(y) B=g(y B) \subseteq g\left(Y_{1}\right)=f_{1}\left(Y_{1}\right)\right) \subseteq X, \\
h(g(y)) B=h(g(y) B) \subseteq h(X)=0 .
\end{gathered}
$$

Поэтому $h(g(y)) \in \operatorname{Sing}(M / X)=0$ и $g(y) \in \operatorname{Ker}(h)=X$.

Так как $g(Y) \subseteq X$, то $g$ индуцирует гомоморфизм $f: Y \rightarrow X$. Поэтому модуль $X$ инъективен относительно $Y$.

2) Обозначим $X=\operatorname{Sing}(M)$. Так как $A$ - несингулярное справа кольцо, то модуль $M / X$ несингулярен; см. [4; Proposition 1.23(a)]. Так как модуль $X$ сингулярен и модуль $Y$ несингулярен, то по 1) модуль $X$ инъективен относительно $Y$.

3) Так как $X$ и $Y$ - замкнутые подмодули несингулярного модуля $M$, то из [4; Proposition 1.23(a)] вытекает, что модули $M / X$ и $M / Y$ несингулярны. По 1) $X$ инъективен относительно $Y$ и модуль $Y$ инъективен относительно $X$.

Лемма 6. Пусть А - строго первичное справа кольцо.

1) Если $X$ - правый $A$-модуль и $X$ инбективен относительно некоторого ненулевого правого идеала кольца $A$, то $X$ - ингективный модуль.

2) Если $X$ - правый $A$-модуль и $X$ инбективен относительно некоторого правого $A$-модуля $Y$, не являющегося существенным расширением сингулярного модуля, то $X-$ инбективный модуль.

3) Если $M$ - автоморфизм-продолжаемый правый $A$-модуль, не являющийся сингулярным, то $M=X \oplus Y$, где $X$ - ингективный сингулярный модуль и $Y$ - ненулевой несингулярный автоморфизм-продолжаемьй модуль, причем либо модуль $Y$ равномерен, либо $Y$ - инбективный неравномерный модуль.

4) Если $M$ - автоморфизм-инвариантный правый A-модуль, не являющийся сингулярным, то $M-$ ингективный модуль. 
Доказательство. 1) Так как модуль $X$ инъективен относительно некоторого ненулевого правого идеала $B$, то по лемме $3(1)$ модуль $X$ инъективен относительно некоторого ненулевого идеала $A B$. Поскольку кольцо $A$ строго первично справа, то идеал $A B$ содержит такое конечное подмножество $\left\{y_{1}, \ldots, y_{n}\right\}$, что $r\left(\left\{y_{1}, \ldots, y_{n}\right\}\right)=0$. По лемме $3(2)$ модуль $X$ инъективен.

2) По лемме 4 существует такой ненулевой правый идеал $B$ кольца $A$, что модуль $B_{A}$ изоморфен подмодулю модуля $Y$. Так как модуль $X$ инъективен относительно модуля $Y$, то по лемме $1(1)$ модуль $X$ инъективен.

3) Обозначим $X=\operatorname{Sing}(M)$. Строго первичное справа кольцо $A$ несингулярно справа; см. [6]. Тогда модуль $M / X$ несингулярен; см. [4; Proposition 1.23(a)]. Так как кольцо $A$ несингулярно справа и модуль $M_{A}$ не является сингулярным, то $M$ не является существенным расширением сингулярного модуля $X$. Поэтому $X \cap Y^{\prime}=0$ для некоторого несингулярного подмодуля $Y^{\prime}$ в $M$. По лемме 5(2) модуль $X$ инъективен относительно $Y^{\prime}$. По 2) $X$ - инъективный модуль. Поэтому $M=$ $X \oplus Y$, где $X$ - инъективный сингулярный модуль и $Y$ - ненулевой несингулярный автоморфизм-продолжаемый модуль. Если модуль $Y$ равномерен, то все доказано.

Допустим, что $Y$ - неравномерный модуль. Тогда существуют такие ненулевые замкнутые подмодули $Y_{1}$ и $Y_{2}$ в $Y$, что $Y_{1} \cap Y_{2}=0$ и $Y_{1} \oplus Y_{2}$ - существенный подмодуль в $Y$. Так как $Y_{1}$ и $Y_{2}$ - замкнутые подмодули несингулярного модуля $Y$, то модули $M / Y_{1}$ и $M / Y_{2}$ несингулярны. По лемме $5(1)$ модуль $Y_{1}$ инъективен относительно ненулевого несингулярного модуля $Y_{2}$ и модуль $Y_{2}$ инъективен относительно ненулевого несингулярного модуля $Y_{1}$. По 2) модули $Y_{1}$ и $Y_{2}$ инъективны. Тогда $Y$ - существенное расширение инъективного модуля $Y_{1} \oplus Y_{2}$. Поэтому $Y=Y_{1} \oplus Y_{2}, Y$ - инъективный неравномерный модуль.

4) По замечанию 4 автоморфизм-инвариантный модуль $M$ является автоморфизм-продолжаемым модулем. Из 3) следует, что $M=X \oplus Y$, где $X$ - инъективный сингулярный модуль и $Y$ - ненулевой несингулярный автоморфизм-инвариантный модуль, причем либо $Y$ - инъективный неравномерный модуль, либо модуль $Y$ равномерен. Достаточно рассмотреть случай, когда $Y$ - ненулевой равномерный автоморфизм-инвариантный модуль. Так как $Y$ - равномерный автоморфизм-инвариантный модуль, то модуль $Y$ инъективен относительно себя; см. [1; Lemma 3.5]. По 2) модуль $Y$ инъективен. Тогда модуль $M$ инъективен.

Замечание 5. Теорема 1 вытекает из леммы 6(4).

\section{Список литературы}

1. Alahmadi A., Er N., Jain S.K., "Modules which are invariant under monomorphisms of their injective hulls", J. Australian Math. Soc., 79:3 (2005), 349-360.

2. Dickson S.E., Fuller K.R., "Algebras for which every indecomposable right module is invariant in its injective envelope", Pacific J. Math., 31:3 (1969), 655-658.

3. Er N., Singh S., Srivastava A.K., "Rings and modules which are stable under automorphisms of their injective hulls", J. Algebra, 379 (2013), 223-229.

4. Goodearl K.R., Ring Theory: Nonsingular Rings and Modules, Marcel Dekker, New York, 1976, $224 \mathrm{pp}$.

5. Guil Asensio P.A., Srivastava A.K., J. Algebra, 388 (2013), 101-106.

6. Handelman D., Lawrence J., "Strongly prime rings", Trans. Amer. Math. Soc., 211 (1975), 209-223. 
7. Jain S.K., Singh S., "Quasi-injective and pseudo-injective modules", Canadian Math. Bull., 18:3 (1975), 359-366.

8. Lawrence J., "A singular primitive ring", Trans. Amer. Math. Soc., 45:1 (1974), 59-62.

9. Lee T.K., Zhou Y., "Modules which are invariant under automorphisms of their injective hulls", J. Algebra Appl., 12:2 (2013).

10. Singh S., Srivastava A.K., "Rings of invariant module type and automorphism-invariant modules", Contemporary Mathematics, Amer. Math. Soc., 609 (2014), 299-311.

11. Tuganbaev A.A., Semidistributive Modules and Rings, Kluwer Academic Publishers, Dordrecht-Boston-London, 1998.

12. Туганбаев А.А., “Автоморфизмы подмодулей и их продолжение”, Дискрет. матем., 25:1 (2013), 144-151.

13. Туганбаев А.А., "Характеристические подмодули инъективных модулей”, Дискрет. матем., 25:2 (2013), 85-90.

14. Wisbauer R., Foundations of Module and Ring Theory, Gordon and Breach, Philadelphia, 1991, 616 pp.

Статья поступила 06.12.2013. 